\title{
A best proximity point theorem for special generalized proximal $\beta$-quasi contractive mappings
}

\author{
M. ladh Ayari', M.M.M. Jaradat ${ }^{2}$ and Z. Mustafa ${ }^{2 *}$
}

${ }^{\text {*Correspondence: zead@qu.edu.qa }}$
${ }^{2}$ Department of Mathematics,
Statistics and Physics, Qatar
University, Doha, Qatar
Full list of author information is
available at the end of the article

available at the end of the article

\begin{abstract}
In this paper, we obtain some best proximity point results for a new class of non-self mappings $T: A \rightarrow B$ called special generalized proximal $\beta$-quasi contractive. Our result is illustrated by an example. Several consequences are derived.

MSC: $47 \mathrm{H} 10 ; 54 \mathrm{H} 25$

Keywords: Best proximity points; Special generalized proximal $\beta$-quasi contractive mappings
\end{abstract}

\section{Introduction}

The famous Banach contraction principle guarantees the existence and uniqueness of fixed points of self-mappings $T: X \longrightarrow X$, where $(X, d)$ is a complete metric space. The Banach contraction principle has been generalized in different ways as in [1]. The main interesting studies deal with the extension of Banach's contraction to non-self-mappings $T: A \longrightarrow B$, where $(A, B)$ is a pair of subsets of a metric space $(X, d)$. In fact such mappings do not necessarily have fixed points. The idea is to look for points where $d(\zeta, T \zeta)=d(A, B)$. Such points are called best proximity points. In 1969, a best approximation theorem was introduced by Fan [2]. Later on, Sadiq Basha [3] proposed necessary and sufficient conditions for the existence of proximal contractions of first and second kind for such points. Several variants of non-self-contractions for the existence of a best proximity point were studied in [4-7].

In 2014, Almeida et al. [8], by using the notion of P-property (weak P-property), proved that some late results about the existence and uniqueness of best proximity points can be obtained from the versions of associated existing results in the fixed point theory.

Our work focuses on the best proximity point theorem for a new family of non-selfmappings called special generalized proximal $\beta$-quasi contractive mappings. As an application to the self-mapping case, the present work generalizes several existing results on fixed point theory as the Banach contraction principle [9] and the generalization of such a principle by Ćirić in [1].

The paper is divided into five sections. Section 2 introduces the notation used herein, presents some definitions, and recalls some useful results. The best proximity point the-

(c) The Author(s) 2019. This article is distributed under the terms of the Creative Commons Attribution 4.0 International License (http://creativecommons.org/licenses/by/4.0/), which permits unrestricted use, distribution, and reproduction in any medium, provided you give appropriate credit to the original author(s) and the source, provide a link to the Creative Commons license, and indicate if changes were made. 
orem with its proof is stated in Sect. 3. Finally, several consequences on the existence and uniqueness of best proximity points and fixed point results are given in Sect. 4.

\section{Preliminaries and definitions}

Let $(A, B)$ be a pair of nonempty subsets of a metric space $(X, d)$. Throughout this work we consider the following notations:

$$
\begin{aligned}
& d(A, B):=\inf \{d(a, b): a \in A, b \in B\} \\
& A_{0}:=\{a \in A: \text { there exists } b \in B \text { such that } d(a, b)=d(A, B)\} \\
& B_{0}:=\{b \in B: \text { there exists } a \in A \text { such that } d(a, b)=d(A, B)\} .
\end{aligned}
$$

Definition 2.1 ([3]) Let $T: A \rightarrow B$ be a mapping. An element $x^{*}$ is said to be a best proximity point of $T$ if $d\left(x^{*}, T x^{*}\right)=d(A, B)$.

Definition 2.2 ([10]) Let $\beta \in(0,+\infty)$. A $\beta$-comparison function is a map $\varphi:[0,+\infty) \rightarrow$ $[0,+\infty)$ satisfying the following properties:

(1) $\varphi$ is nondecreasing;

(2) $\lim _{n \rightarrow \infty} \varphi_{\beta}^{n}(t)=0$ for all $t>0$, where $\varphi_{\beta}^{n}$ denotes the $n$th iterate of $\varphi_{\beta}$ and $\varphi_{\beta}(t)=\varphi(\beta t)$

(3) there exists $s \in(0,+\infty)$ such that $\sum_{n=1}^{\infty} \varphi_{\beta}^{n}(s)<\infty$.

The set of all $\beta$-comparison functions $\varphi$ satisfying (1), (2), and (3) will be denoted by $\Phi_{\beta}$.

Remark 2.3 Let $\alpha, \beta \in(0,+\infty)$. If $\alpha<\beta$, then $\Phi_{\beta} \subset \Phi_{\alpha}$.

A useful lemma concerning the comparison functions $\Phi_{\beta}$ was performed in [10].

Lemma 2.4 ([10]) Let $\beta \in(0,+\infty)$ and $\varphi \in \Phi_{\beta}$. Then

(1) $\varphi_{\beta}$ is nondecreasing;

(2) $\varphi_{\beta}(t)<t$ for all $t>0$;

(3) $\sum_{n=1}^{\infty} \varphi_{\beta}^{n}(t)<\infty$ for all $t>0$.

Definition 2.5 ([11]) Let $(A, B)$ be a pair of nonempty subsets of a metric space $(X, d)$ such that $A_{0}$ is nonempty. Then the pair $(A, B)$ is said to have the P-property iff $d\left(\zeta_{1}, \eta_{1}\right)=$ $d\left(\zeta_{2}, \eta_{2}\right)=d(A, B) \Longrightarrow d\left(\zeta_{1}, \zeta_{2}\right)=d\left(\eta_{1}, \eta_{2}\right)$, where $\zeta_{1}, \zeta_{2} \in A$ and $\eta_{1}, \eta_{2} \in B$.

Definition 2.6 We say that $B$ is approximately compact with respect to $A$ iff every sequence $\left\{\eta_{n}\right\} \subset B$ satisfying $\lim _{n \rightarrow+\infty} d\left(\zeta, \eta_{n}\right)=d(\zeta, B)$ for some $\zeta \in A$ has a convergent subsequence.

\section{Main results and theorems}

First, we introduce the following concept.

Definition 3.1 Let $(X, d)$ be a metric space and $(A, B)$ be a pair of nonempty subsets of $X$. Let $\beta \in(0,+\infty)$. A non-self-mapping $T: A \rightarrow B$ is said to be a special generalized proximal $\beta$-quasi contractive mapping iff there exist $\varphi \in \Phi_{\beta}$ and positive numbers $\alpha_{i}, i=1,2,3,4$, such that:

$$
d(T \zeta, T \eta) \leq \varphi\left(M_{T}(\zeta, \eta)\right), \quad \forall \zeta, \eta \in A,
$$


where

$$
\begin{aligned}
M_{T}(\zeta, \eta)= & \max \left\{\alpha_{0} d(\zeta, \eta), \alpha_{1}(d(\zeta, T \zeta)-d(A, B)),\right. \\
& \alpha_{2}(d(\eta, T \eta)-d(A, B)), \alpha_{3}(d(\eta, T \zeta)-d(A, B)), \\
& \left.\alpha_{4}(d(\zeta, T \eta)-d(A, B))\right\} .
\end{aligned}
$$

$M_{T}(\zeta, \eta)$ was introduced in [12]. Our main result is given by the following best proximity point theorem.

Theorem 3.2 Let $(A, B)$ be a pair of nonempty closed subsets of a complete metric space $(X, d)$ such that $A_{0}$ is nonempty. Consider a non-self-mapping $T: A \longrightarrow B$ satisfying the following conditions:

(1) $T\left(A_{0}\right) \subset B_{0}$ and the pair $(A, B)$ satisfies the P-property.

(2) $B$ is approximately compact with respect to $A$.

(3) There exist elements $\zeta_{0}, \zeta_{1} \in A$ such that

$$
d\left(\zeta_{1}, T \zeta_{0}\right)=d(A, B)
$$

(4) There exists $\beta \geq \max _{0 \leq k \leq 3}\left\{\alpha_{k}, 2 \alpha_{4}\right\}$ such that $T$ is special generalized proximal $\beta$-quasi contractive.

Moreover, assume that one of the following conditions holds:

- $\varphi$ is continuous;

- $\beta>\max \left\{\alpha_{1}, \alpha_{3}\right\}$

Then $T$ has a unique best proximity point $\zeta \in A$ such that $d(\zeta, T \zeta)=d(A, B)$.

Proof From condition (3), there exist $\zeta_{0}, \zeta_{1} \in A$ such that $d\left(\zeta_{1}, T \zeta_{0}\right)=d(A, B)$. Then $A_{0} \neq \phi$, $B_{0} \neq \phi$. Now $T\left(\zeta_{1}\right) \in B_{0}$ since $T\left(A_{0}\right) \subset B_{0}$, so there exists $\zeta_{2} \in A$ such that $d\left(\zeta_{2}, T \zeta_{1}\right)=$ $d(A, B)$. Continuing this process, we can build a sequence $\left\{\zeta_{n}\right\} \subset A_{0}$ such that

$$
d\left(\zeta_{n+1}, T \zeta_{n}\right)=d(A, B) \quad \text { for all } n \in \mathbb{N} \cup\{0\}
$$

Using the P-property, we get

$$
d\left(\zeta_{n}, \zeta_{n+1}\right)=d\left(T \zeta_{n-1}, T \zeta_{n}\right)
$$

Our next step is to prove that $\left\{\zeta_{n}\right\}$ is a Cauchy sequence.

Since $T$ is special generalized proximal $\beta$-quasi contractive, we obtain

$$
d\left(\zeta_{n}, \zeta_{n+1}\right)=d\left(T \zeta_{n-1}, T \zeta_{n}\right) \leq \varphi\left(M_{T}\left(\zeta_{n-1}, \zeta_{n}\right)\right), \quad \forall n \in \mathbb{N}
$$

On the other hand, using (3.2), (3.3), and the triangular inequality, we get

$$
\begin{aligned}
M_{T}\left(\zeta_{n-1}, \zeta_{n}\right)= & \max \left\{\alpha_{0} d\left(\zeta_{n-1}, \zeta_{n}\right), \alpha_{1}\left(d\left(\zeta_{n-1}, T \zeta_{n-1}\right)-d(A, B)\right),\right. \\
& \alpha_{2}\left(d\left(\zeta_{n}, T \zeta_{n}\right)-d(A, B)\right), \alpha_{3}\left(d\left(\zeta_{n}, T \zeta_{n-1}\right)-d(A, B)\right), \\
& \left.\alpha_{4}\left(d\left(\zeta_{n-1}, T \zeta_{n}\right)-d(A, B)\right)\right\}
\end{aligned}
$$




$$
\begin{aligned}
= & \max \left\{\alpha_{0} d\left(\zeta_{n-1}, \zeta_{n}\right), \alpha_{1}\left(d\left(\zeta_{n-1}, T \zeta_{n-1}\right)-d(A, B)\right),\right. \\
& \left.\alpha_{2}\left(d\left(\zeta_{n}, T \zeta_{n}\right)-d(A, B)\right), \alpha_{4}\left(d\left(\zeta_{n-1}, T \zeta_{n}\right)-d(A, B)\right)\right\} \\
\leq & \max \left\{\alpha_{0} d\left(\zeta_{n-1}, \zeta_{n}\right), \alpha_{1} d\left(\zeta_{n-1}, \zeta_{n}\right), \alpha_{2} d\left(\zeta_{n}, \zeta_{n+1}\right),\right. \\
& \left.\alpha_{4} d\left(\zeta_{n-1}, \zeta_{n}\right)+\alpha_{4} d\left(\zeta_{n}, \zeta_{n+1}\right)\right\} \\
\leq & \beta \max \left\{d\left(\zeta_{n-1}, \zeta_{n}\right), d\left(\zeta_{n}, \zeta_{n+1}\right)\right\} .
\end{aligned}
$$

Hence,

$$
M_{T}\left(\zeta_{n-1}, \zeta_{n}\right) \leq \beta \max \left\{d\left(\zeta_{n-1}, \zeta_{n}\right), d\left(\zeta_{n}, \zeta_{n+1}\right)\right\}
$$

where $\beta \geq \max _{0 \leq k \leq 3}\left\{\alpha_{k}, 2 \alpha_{4}\right\}$. Using inequalities (3.3) and (3.5) and taking into consideration the fact that $\varphi$ is nondecreasing, we get that

$$
d\left(\zeta_{n+1}, \zeta_{n}\right) \leq \varphi\left(\beta \max \left\{d\left(\zeta_{n-1}, \zeta_{n}\right), d\left(\zeta_{n}, \zeta_{n+1}\right)\right\}\right)=\varphi_{\beta}\left(\max \left\{d\left(\zeta_{n-1}, \zeta_{n}\right), d\left(\zeta_{n}, \zeta_{n+1}\right)\right\}\right)
$$

Suppose that, for some $n$, we have $d\left(\zeta_{n-1}, \zeta_{n}\right) \leq d\left(\zeta_{n}, \zeta_{n+1}\right)$. It follows that $d\left(\zeta_{n+1}, \zeta_{n}\right) \leq$ $\varphi_{\beta}\left(d\left(\zeta_{n+1}, \zeta_{n}\right)\right)<d\left(\zeta_{n+1}, \zeta_{n}\right)$, which is a contradiction.

Then, for all $n \geq 0$, we necessary have $d\left(\zeta_{n-1}, \zeta_{n}\right)>d\left(\zeta_{n}, \zeta_{n+1}\right)$, and it follows that

$$
d\left(\zeta_{n+1}, \zeta_{n}\right) \leq \varphi_{\beta}\left(d\left(\zeta_{n-1}, \zeta_{n}\right)\right), \quad \forall n \in \mathbb{N}
$$

Then, by induction, we obtain that

$$
d\left(\zeta_{n+1}, \zeta_{n}\right) \leq \varphi_{\beta}^{n}\left(d\left(\zeta_{1}, \zeta_{0}\right)\right), \quad \forall n \in \mathbb{N} \cup\{0\}
$$

Let $\epsilon>0$ be fixed. Since the numerical series $\sum_{n=1}^{+\infty} \varphi_{\beta}^{n}\left(d\left(\zeta_{1}, \zeta_{0}\right)\right)$ converges, there exists a positive integer $N$ such that $\sum_{n \geq N}^{+\infty} \varphi_{\beta}^{n}\left(d\left(\zeta_{1}, \zeta_{0}\right)\right)<\epsilon$. For $m>n>N$, using the triangular inequality, the convergence of the series, and (3.7), we obtain

$$
\begin{aligned}
d\left(\zeta_{n}, \zeta_{m}\right) & \leq \sum_{k=n}^{m-1} d\left(\zeta_{k}, \zeta_{k+1}\right) \\
& \leq \sum_{k=n}^{m-1} \varphi_{\beta}^{k}\left(d\left(\zeta_{1}, \zeta_{0}\right)\right)
\end{aligned}
$$

since the series $\sum_{n=1}^{+\infty} \varphi_{\beta}^{n}(t)$ converges for all $t \geq 0$; as a result

$$
\sum_{k=n}^{m-1} \varphi_{\beta}^{k}\left(d\left(\zeta_{1}, \zeta_{0}\right)\right) \longrightarrow 0 \quad \text { as } n, m \longrightarrow+\infty
$$

Therefore, $\zeta_{n}$ is a Cauchy sequence. Since $(X, d)$ is complete and $A$ is closed, then the sequence $\left\{\zeta_{n}\right\}$ converges to some element $\zeta \in A$.

On the other hand, we have

$$
\begin{aligned}
d(\zeta, B) & \leq d\left(\zeta, T \zeta_{n}\right) \\
& \leq d\left(\zeta, \zeta_{n+1}\right)+d\left(\zeta_{n+1}, T \zeta_{n}\right)
\end{aligned}
$$




$$
\begin{aligned}
& =d\left(\zeta, \zeta_{n+1}\right)+d(A, B) \\
& \leq d\left(\zeta, \zeta_{n+1}\right)+d(\zeta, B)
\end{aligned}
$$

As $n \rightarrow \infty$, we get that the sequence $d\left(\zeta, T \zeta_{n}\right)$ converges to $d(\zeta, B)$. Using hypothesis (2) of the theorem, there exists a subsequence $\left\{\zeta_{n(k)}\right\}$ of $\left\{\zeta_{n}\right\}$ such that $T\left(\zeta_{n(k)}\right)$ converges to some $\eta \in B$. Hence

$$
\begin{aligned}
d(A, B) & \leq d(\zeta, \eta) \\
& \leq d\left(\zeta, \zeta_{n_{k}+1}\right)+d\left(\zeta_{n_{k}+1}, T \zeta_{n_{k}}\right)+d\left(T \zeta_{n_{k}}, \eta\right) \\
& =d\left(\zeta, \zeta_{n_{k}+1}\right)+d(A, B)+d\left(T \zeta_{n_{k}}, \eta\right) .
\end{aligned}
$$

As $n \rightarrow \infty$, we get that $d(\zeta, \eta)=d(A, B)$ and therefore $\zeta \in A_{0}$. Since $T\left(A_{0}\right) \subset B_{0}$, there exists $u \in A$ such that $d(u, T \zeta)=d(A, B)$. Consequently, we obtain $d(u, T \zeta)=d\left(\zeta_{n+1}, T \zeta_{n}\right)=$ $d(A, B)$. Using the P-property, we deduce that $d\left(u, \zeta_{n+1}\right)=d\left(T \zeta, T \zeta_{n}\right)$.

Since $T$ is special generalized $\beta$-proximal quasi-contractive, we obtain

$$
\begin{aligned}
d\left(u, \zeta_{n+1}\right) & =d\left(T \zeta, T \zeta_{n}\right) \\
& \leq \varphi\left(M_{T}\left(\zeta, \zeta_{n}\right)\right), \quad \forall n \in \mathbb{N}
\end{aligned}
$$

where

$$
\begin{aligned}
M_{T}\left(\zeta, \zeta_{n}\right)= & \max \left\{\alpha_{0} d\left(\zeta, \zeta_{n}\right), \alpha_{1}(d(\zeta, T \zeta)-d(A, B)),\right. \\
& \alpha_{2}\left(d\left(\zeta_{n}, T \zeta_{n}\right)-d(A, B)\right), \alpha_{3}\left(d\left(\zeta_{n}, T \zeta\right)-d(A, B)\right), \\
& \left.\alpha_{4}\left(d\left(\zeta, T \zeta_{n}\right)-d(A, B)\right)\right\}
\end{aligned}
$$

On the other hand, using the triangular inequality and (3.2), we have

$$
\begin{aligned}
M_{T}\left(\zeta, \zeta_{n}\right) \leq & \max \left\{\alpha_{0} d\left(\zeta, \zeta_{n}\right), \alpha_{1}(d(\zeta, T \zeta)-d(A, B))\right. \\
& \alpha_{2} d\left(\zeta_{n}, \zeta_{n+1}\right), \alpha_{3}\left(d\left(\zeta_{n}, \zeta\right)+d(\zeta, T \zeta)-d(A, B)\right), \\
& \left.\alpha_{4} d\left(\zeta, \zeta_{n+1}\right)\right\}
\end{aligned}
$$

Moreover, using the triangular inequality, we get

$$
\begin{aligned}
d(\zeta, T \zeta) & \leq d\left(\zeta, \zeta_{n+1}\right)+d\left(\zeta_{n+1}, T \zeta_{n}\right)+d\left(T \zeta_{n}, T \zeta\right) \\
& =d\left(\zeta, \zeta_{n+1}\right)+d(A, B)+d\left(T \zeta_{n}, T \zeta\right), \quad \forall n \in \mathbb{N} .
\end{aligned}
$$

Using inequality (3.11) and (3.2), we obtain that

$$
\begin{array}{r}
d(\zeta, T \zeta)-d\left(\zeta, \zeta_{n+1}\right)-d(A, B) \\
\leq \varphi\left(M_{T}\left(\zeta_{n}, \zeta\right)\right), \quad \forall n \in \mathbb{N} .
\end{array}
$$


Let $s=d(\zeta, T \zeta)-d(A, B)$, letting $n \longrightarrow+\infty$ in inequality (3.10), we get

$$
\lim _{n \rightarrow+\infty} M_{T}\left(\zeta_{n}, \zeta\right) \leq \max \left\{\alpha_{1}, \alpha_{3}\right\} s
$$

Suppose that $s>0$. If $\varphi$ is continuous, letting $n \longrightarrow+\infty$ in inequality (3.12) and using the fact that $\varphi$ is nondecreasing, we get

$$
s \leq \varphi\left(\max \left\{\alpha_{1}, \alpha_{3}\right\} s\right) \leq \varphi(\beta s)=\varphi_{\beta}(s)<s,
$$

which is a contradiction. If $\beta>\max \left\{\alpha_{1}, \alpha_{3}\right\}$. We claim that also $s=0$. Suppose that $s>0$. Using inequality (3.13) and the definition of the limit, there exist $\varepsilon>0$ and $N>0$ such that, for all $n>N$, we have $M_{T}\left(\zeta_{n}, \zeta\right)<\left(\max \left\{\alpha_{1}, \alpha_{3}\right\}+\varepsilon\right) s$. Since $\varphi$ is nondecreasing, from (3.13), we get

$$
\begin{aligned}
d(\zeta, T \zeta)-d\left(\zeta, \zeta_{n+1}\right)-d(A, B) & \leq \varphi\left(M_{T}\left(\zeta_{n}, \zeta\right)\right) \\
& \leq \varphi\left(\left(\max \left\{\alpha_{1}, \alpha_{3}\right\}+\varepsilon\right) s\right) \\
& =\varphi_{\beta}\left(\frac{\max \left\{\alpha_{1}, \alpha_{3}\right\}+\varepsilon}{\beta} s\right) \\
& <\frac{\max \left\{\alpha_{1}, \alpha_{3}\right\}+\varepsilon}{\beta} s<s
\end{aligned}
$$

By letting $n \longrightarrow+\infty$ in (3.14), we get

$$
s<\frac{\max \left\{\alpha_{1}, \alpha_{3}\right\}+\varepsilon}{\beta} s<s,
$$

which is a contradiction. Therefore $s=0$ and so $d(\zeta, T \zeta)-d(A, B)=0$, which implies that $d(\zeta, T \zeta)=d(A, B)$, that is, $\zeta$ is the best proximity point.

For the uniqueness, suppose that there are two distinct best proximity points $\zeta$ and $\eta$ such that $d(\zeta, T \zeta)=d(\eta, T \eta)=d(A, B)$. Using the P-property, we get $d(\zeta, \eta)=d(T \zeta, T \eta)$ On the other hand, since $T$ is special generalized proximal $\beta$-quasi contractive, we deduce that

$$
d(\zeta, \eta)=d(T \zeta, T \eta) \leq \varphi\left(M_{T}(\zeta, \eta)\right)
$$

where

$$
M_{T}(\zeta, \eta)=\max \left\{\alpha_{0} d(\zeta, \eta), \alpha_{3}(d(\eta, T \zeta)-d(A, B)), \alpha_{4}(d(\zeta, T \eta)-d(A, B))\right\}
$$

Using triangular inequalities in $M_{T}(\zeta, \eta)$ and the fact that $\zeta$ and $\eta$ are best proximity points of $T$, we get

$$
M_{T}(\zeta, \eta) \leq\left\{\alpha_{0}, \alpha_{3}, \alpha_{4}\right\} d(\zeta, \eta)
$$

Let $r=d(\zeta, \eta)$. Since $\varphi$ is nondecreasing, we obtain

$$
r \leq \varphi\left(\left\{\alpha_{0}, \alpha_{3}, \alpha_{4}\right\} r\right) \leq \varphi(\beta r)=\varphi_{\beta}(r)<r
$$

which is a contradiction. 
Example 3.3 Consider the complete metric space $X=\mathbb{R}$ with the metric $d(\zeta, \eta)=|\zeta-\eta|$. Let $A=[0,2]$ and $B=[4,6]$. These sets are closed on the metric space $(X, d)$. Also, since $B$ is compact, then $B=[4,6]$ is approximately compact with respect to $A=[0,2]$. Also, let $T: A \longrightarrow B$ be defined by $T(\zeta)=6-\zeta$. Then it is easy to see that $d(A, B)=2$ and $A_{0}=\{2\}$, $B_{0}=\{4\}$. Thus, $T\left(A_{0}\right)=T(\{2\})=\{4\}=B_{0}$. Now we shall show that $T$ is special generalized proximal $\beta$-quasi contractive with $\phi(t)=\frac{1}{2} t, \beta=2$, and $\alpha_{1}=2$ and $\alpha_{i}=0$ for $i=1,2,3,4$. Note that $\phi(t)=\frac{1}{2} t \in \Phi_{2} \subset \Phi_{1}$ using Remark 2.3.

$$
0=d(T \zeta, T \eta)=|\zeta-\eta| \leq \frac{1}{2} \max \{2 d(\zeta, \eta), 0,0,0,0\} .
$$

The function $\phi(t)$ is continuous mappings as well as $\beta=2>\max \left\{\alpha_{1}, \alpha_{3}\right\}=0$. We deduce, using our Theorem 3.2, that $T$ has a unique best proximity point which is $\zeta_{*}=2$ in this example.

$$
d\left(\zeta_{*}, T \zeta_{*}\right)=d(2, T(2))=d(2,4)=d(A, B)=2 .
$$

\section{Consequences}

Several consequences of the main results of Sect. 3 are established next.

First, as an application to best proximity points, we propose the following results, which are an immediate consequence of our main Theorem 3.2.

Corollary 4.1 Let $(A, B)$ be a pair of nonempty closed subsets of a complete metric space $(X, d)$ such that $A_{0}$ is nonempty. Consider a non-self-mapping $T: A \longrightarrow B$ satisfying the following conditions:

(1) $T\left(A_{0}\right) \subset B_{0}$ and the pair $(A, B)$ satisfies the P-property.

(2) $B$ is approximately compact with respect to $A$.

(3) There exist elements $\zeta_{0}, \zeta_{1} \in A$ such that

$$
d\left(\zeta_{1}, T \zeta_{0}\right)=d(A, B)
$$

(4) There exists $\varphi \in \Phi_{2}$ such that

$$
d(T \zeta, T \eta) \leq \varphi(M(\zeta, \eta)), \quad \forall \zeta, \eta \in A
$$

where

$$
\begin{aligned}
M(\zeta, \eta)= & \max \{d(\zeta, \eta), d(\zeta, T \zeta)-d(A, B)) \\
& \left.d(\eta, T \eta)-d(A, B), \frac{d(\eta, T \zeta)+d(\zeta, T \eta)}{2}-d(A, B)\right\} .
\end{aligned}
$$

Then $T$ has the unique best proximity point $\zeta \in A$ such that $d(\zeta, T \zeta)=d(A, B)$.

Proof Note that the above quantity $M(\zeta, \eta)$ was introduced by Jleli, Karapinar, and Samet in [13].

The main idea is that

$$
M(\zeta, \eta) \leq M_{T}(\zeta, \eta)
$$


where

$$
\begin{aligned}
M_{T}(\zeta, \eta)= & \max \{d(\zeta, \eta), d(\zeta, T \zeta)-d(A, B)), \\
& d(\eta, T \eta)-d(A, B), d(\eta, T \zeta)-d(A, B), d(\zeta, T \eta)-d(A, B)\} .
\end{aligned}
$$

So $\alpha_{i}=1$ for all $i \in\{0,1, \ldots, 4\}$. So for $\beta \geq 2>\max \left\{\alpha_{1}, \alpha_{3}\right\}=1$. According to our Theorem 3.2, if the comparison function $\varphi \in \Phi_{2}$, then $T$ has a unique proximity point in $A$.

Corollary 4.2 Let $(A, B)$ be a pair of nonempty closed subsets of a complete metric space $(X, d)$ such that $A_{0}$ is nonempty. Consider a non-self-mapping $T: A \longrightarrow B$ satisfying the following conditions:

(1) $T\left(A_{0}\right) \subset B_{0}$ and the pair $(A, B)$ satisfies the P-property.

(2) $B$ is approximately compact with respect to $A$.

(3) There exist elements $\zeta_{0}, \zeta_{1} \in A$ such that

$$
d\left(\zeta_{1}, T \zeta_{0}\right)=d(A, B)
$$

(4) There exists $q \in[0,1)$ such that

$$
d(T \zeta, T \eta) \leq q M(\zeta, \eta), \quad \forall \zeta, \eta \in A,
$$

where

$$
\begin{aligned}
M(\zeta, \eta)= & \max \{d(\zeta, \eta), d(\zeta, T \zeta)-d(A, B), \\
& d(\eta, T \eta)-d(A, B), d(\eta, T \zeta)-d(A, B), d(\zeta, T \eta)-d(A, B)\} .
\end{aligned}
$$

Then $T$ has a unique best proximity point $\zeta \in A$ such that $d(\zeta, T \zeta)=d(A, B)$.

Proof Let $\varphi=q t$, which belongs to $\Phi_{1}$ and is continuous. According our Theorem 3.2, $T$ has a unique proximity point in $A$.

Before proposing consequences of our result to the existence and uniqueness of fixed points for self-mappings, we introduce the following definition.

Definition 4.3 Let $A$ be a nonempty set of a metric space $(X, d)$. A self-mapping $T: A \rightarrow A$ is called generalized $\beta$-quasi contractive if there exists a function $\varphi \in \Phi_{\beta}$, where $\beta>0$ such that, for all $\zeta, \eta \in A$, we have

$$
d(T \zeta, T \eta) \leq \varphi\left(M_{T}(\zeta, \eta)\right)
$$

where

$$
M_{T}(\zeta, \eta)=\max \left\{\alpha_{0} d(\zeta, \eta), \alpha_{1} d(\zeta, T \zeta), \alpha_{2} d(\eta, T \eta), \alpha_{3} d(\zeta, T \eta), \alpha_{4} d(\eta, T \zeta)\right\}
$$

with $\alpha_{k} \geq 0$ for $k=0, \ldots, 4$. 
Several papers dealt with fixed point theory in the context of the generalizing of Banach's principle as in [14-20]. By our generalized $\beta$-quasi contractive mapping, we can propose some theorems on the existence and uniqueness of fixed points in complete spaces in a simple way.

Corollary 4.4 Let $(X, d)$ be a nonempty complete metric space. Consider a self-mapping $T: X \rightarrow X$. Suppose that there exists $\beta \geq \max _{0 \leq k \leq 3}\left\{\alpha_{k}, 2 \alpha_{4}\right\}$ such that $T$ is a $\beta$-quasi contractive mapping.

Moreover, assume that one of the following conditions holds:

- $\varphi$ is continuous;

- $\beta>\max \left\{\alpha_{1}, \alpha_{3}\right\}$.

Then $T$ has a unique fixed point in $X$.

Proof This is an immediate consequence of our main Theorem 3.2 since $A=B=X$ and every set is approximately compact with its self. Moreover, the notion of special generalized $\beta$-proximal quasi-contractive on the self-mapping case is exactly a $\beta$-quasi-contractive one.

Also the famous Cirić theorem is an immediate consequence of our theorem.

Corollary 4.5 Let $(X, d)$ be a complete metric space, and let $T: X \rightarrow X$ be a quasicontraction, that is,

$$
\begin{aligned}
& d(T \zeta, T \eta) \leq q \max \{d(\zeta, \eta), d(\zeta, T \zeta), d(\eta, T \eta), d(\zeta, T \eta), d(\eta, T \zeta)\} \\
& \quad \text { for all } \zeta, \eta \in X
\end{aligned}
$$

where $q \in[0,1)$ is some constant. Then $T$ has a unique fixed point $\zeta \in X$.

Proof Using our main Theorem 3.2, since $A=B=X$ and every set is approximately compact with its self, the function $\varphi(t)=q t$, which is continuous and belongs to the set $\Phi_{1}$.

\section{Conclusion}

Improvements to some best proximity point theorems are proposed. This has been achieved by introducing a suitable mapping called special generalized proximal $\beta$-quasi contractive. These are non-self-mappings involving $\beta$-comparison functions. As an application, we establish the existence and uniqueness of well-known fixed point results for the case of self-mappings on complete metric spaces. We confirm our result by a suitable example.

\section{Acknowledgements}

Not applicable.

Funding

Not applicable.

Abbreviations

Not applicable.

Availability of data and materials

Please contact the authors for data requests. 


\section{Competing interests}

The authors declare that they have no competing interests.

\section{Authors' contributions}

The authors contributed equally to the preparation of the paper. The authors read and approved the final manuscript.

\section{Author details}

${ }^{1}$ Carthage University, National Institute of Applied Sciences and Technology of Tunis, Tunis, Tunisie. ${ }^{2}$ Department of

Mathematics, Statistics and Physics, Qatar University, Doha, Qatar.

\section{Publisher's Note}

Springer Nature remains neutral with regard to jurisdictional claims in published maps and institutional affiliations.

Received: 5 April 2019 Accepted: 13 November 2019 Published online: 02 December 2019

\section{References}

1. Ćirić, L.B.: Generalized contractions and fixed point theorems. Publ. Inst. Math. 12, 19-26 (1971)

2. Fan, K.: Extensions of two fixed point theorems of F.E. Brower. Math. Z. 112, 234-240 (1969)

3. Sadi Bacha, S.: Extensions of Banach's contraction principle. Numer. Funct. Anal. Optim. 31, 569-576 (2010)

4. Prolla, J.B.: Fixed point theorems for set-valued mappings and existence of best approximations. Numer. Funct. Anal. Optim. 5, 449-455 (1982/83)

5. Reich, S.: Approximate selections, best approximations, fixed points and invariant sets. J. Math. Anal. Appl. 62, 104-113 (1978)

6. Sehgal, V.M., Singh, S.P.K.: A generalization to multifunctions of Fan's best approximation theorem. Proc. Am. Math. Soc. 102, 534-537 (1988)

7. Sehgal, V.M., Singh, S.P.K.: A theorem of best approximation. Numer. Funct. Anal. Optim. 10, 181-184 (1989)

8. Almeida, A., Karapinar, E., Sadarangani, K.: A note on best proximity point theorems under weak P-property. Abstr. Appl. Anal. 2014, Article ID 716825 (2014)

9. Banach, S.: Sur les opérations dans les ensembles abstraits et leur application aux équations intégrales. Fundam. Math. 3(1), 133-181 (1922)

10. Ayari, M.I., Berzig, M., Kedim, I.: Coincidence and common fixed point results for $\beta$-quasi contractive mappings on metric spaces endowed with binary relation. Math. Sci. 10(3), 105-114 (2016)

11. Raj, V.S.: A best proximity point theorem for weakly contractive non-self-mappings. Nonlinear Anal. 74, 4804-4808 (2011)

12. Ayari, M.I.: Best proximity point theorems for generalized $\alpha$ - $\beta$-proximal quasi-contractive mappings. Fixed Point Theory Appl. 2017, 16 (2017)

13. Jleli, M., Karapinar, E., Samet, B.: Best proximity points for generalized $\alpha$ - $\psi$-proximal contractive type mapping. J. Appl. Math. 2013, Article ID 534127 (2013)

14. Shatanawi, W., Mustafa, Z., Tahat, N.: Some coincidence point theorems for nonlinear contraction in ordered metric spaces. Fixed Point Theory Appl. 2011, 68 (2011). https://doi.org/10.1186/1687-1812-2011-68

15. Mustafa, Z., Aydi, H., Karapinar, E.: Mixed $g$-monotone property and quadruple fixed point theorems in partial ordered metric space. Fixed Point Theory Appl. 2012, 71 (2012). https://doi.org/10.1186/1687-1812-2012-71

16. Shatanawi, W.A., Postolache, M., Mustafa, Z., Taha, N.: Some theorems for Boyd-Wong type contractions in ordered metric spaces. Abstr. Appl. Anal. 2012, Article ID 359054 (2012). https://doi.org/10.1155/2012/359054

17. Karapinar, E., Aydi, H., Mustafa, Z.: Some tripled coincidence point theorems for almost generalized contractions in ordered metric spaces. Tamkang J. Math. 44(3), 233-251 (2013). https://doi.org/10.5556/j.tkjm.44.2013.990

18. Mustafa, Z., Karapinar, E., Aydi, H.: A discussion on generalized almost contractions via rational expressions in partially ordered metric spaces. J. Inequal. Appl. 2014(1), 219 (2014)

19. Mustafa, Z., Huang, H., Radenović, S.: Some remarks on the paper some fixed point generalizations are not real generalizations. J. Adv. Math. Stud. 9(1), 110-116 (2016)

20. Mustafa, Z., Jaradat, M.M.M., Karapinar, E.: A new fixed point result via property P with an application. J. Nonlinear Sci. Appl. 10, 2066-2078 (2017)

\section{Submit your manuscript to a SpringerOpen ${ }^{\circ}$ journal and benefit from:}

- Convenient online submission

- Rigorous peer review

- Open access: articles freely available online

- High visibility within the field

- Retaining the copyright to your article

Submit your next manuscript at $>$ springeropen.com 